\title{
Inappropriate shocks in a patient treated with a cardioverter defibrillator
}

\author{
Wim Hutse, Rene Tavernier, Luc Jordaens
}

Department of Cardiology, University Hospital Ghent, Ghent, Belgium W Hutse

R Tavernier

L Jordaens

Correspondence to: Professor Dr L Jordaens, Department of Cardiology, University Hospital Ghent, De Pintelaan 185, B-9000 Ghent, Belgium.

Accepted for publication 3 October 1996
A 70 year old male patient with an old transmural inferior myocardial infarction suffered monomorphic ventricular tachycardia (VT) during exercise. A 95\% stenosis of the left anterior artery and occlusion of the circumflex artery were observed. The left ventricular ejection fraction was $68 \%$. No residual ischaemia was detected after balloon angioplasty. During electrophysiological testing, ventricular fibrillation (VF) could be induced. A subpectoral cardioverter defibrillator (Medtronic PCD 7218) was implanted. Defibrillation threshold was $3 \mathrm{~J}$, the $\mathrm{R}$ wave $18 \mathrm{mV}$ (fig 1 ). VF therapy was programmed from 180 beats $/ \mathrm{min}$.

Three weeks later, the patient received a shock during a sinus rhythm of 112 beats $/ \mathrm{min}$. In the next 12 hours, he received several shocks during sinus rhythm. On admission, questioning revealed that he had received 19 shocks during 44 episodes of "tachycardia". Stored ECGs of the last three episodes showed
$\mathrm{T}$ wave sensing, leading to detection of VF during sinus rhythm (fig 2).

Sensing parameters were adjusted. During observation only a short episode of atrial fibrillation was seen. Eight hours after admission, he developed cardiogenic shock. A new right bundle branch block and ST segment changes occurred. An echocardiogram showed a dilated right ventricle, with a small, hypokinetic left ventricle. Pulmonary embolism was diagnosed. The patient's status deteriorated within minutes towards cardiac arrest. Massive pulmonary embolism was confirmed on autopsy, with a saddle embolus in the main pulmonary artery.

Implantable cardioverters defibrillators have been associated with a very high complication rate, including inappropriate shocks. ${ }^{12}$ The most important causes are atrial fibrillation, regular supraventricular tachycardia, and nonsustained VT. ${ }^{12}$ Other mechanisms include

Figure 1 Intracardiac $E C G$ at the time of implantation. Appropriate sensing of VF and of sinus rhythm after conversion. The sensitivity was programmed to $0.3 \mathrm{mV}$.

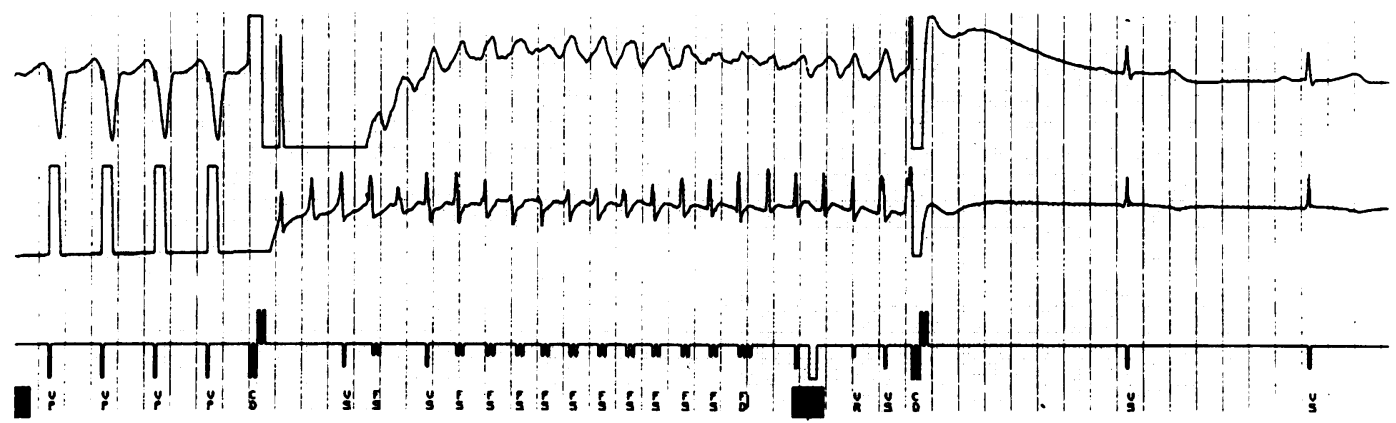

Figure 2 Intracardiac ECG at the time of inappropriate shocks. Event markers show $T$ wave sensing with doublecounting. TS, tachycardia sensed; $F S$, fibrillation sensed; $F D$, fibrillation detected.

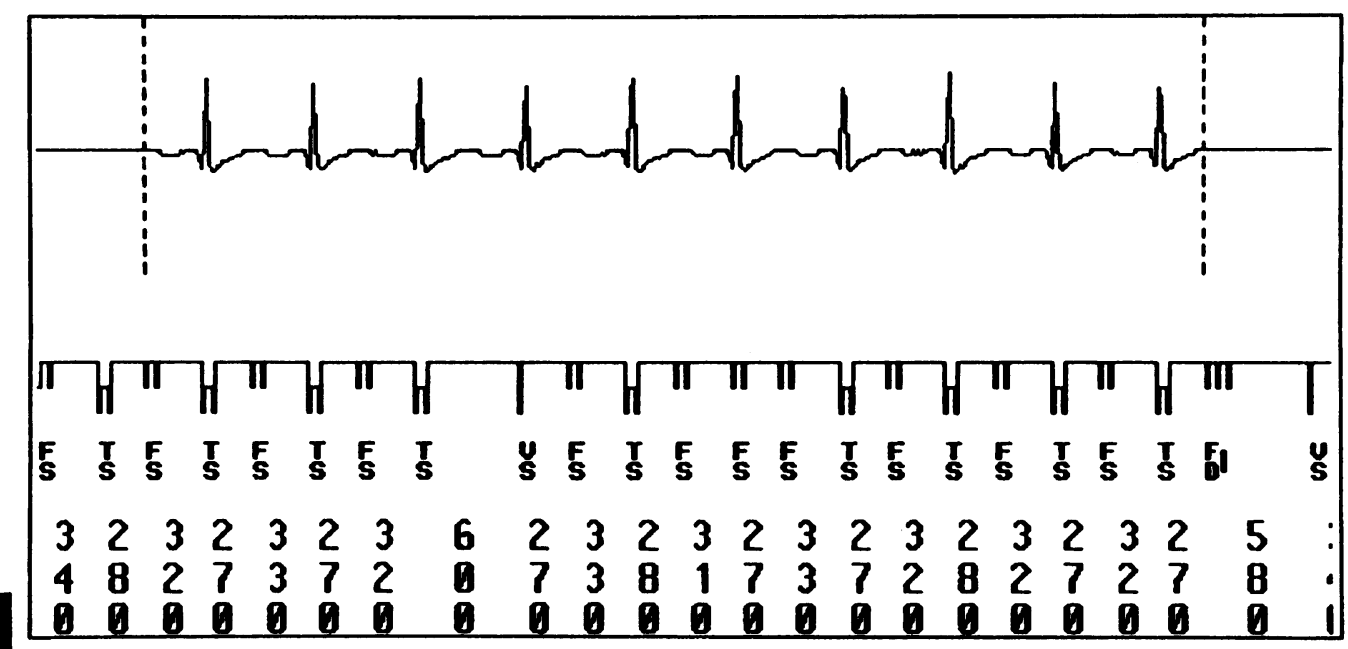


magnet application, pacemaker interference, rate sensing lead malfunction, and oversensing. ${ }^{1}$ Our patient had $\mathrm{T}$ wave sensing with double counting. Lead fracture as a possible cause for shocks during movements can lead to sensing of muscle potentials. Oversensing occurred in one of 24 patients with similar devices. $^{2}$ To our knowledge, this is the first report in which pulmonary embolism as a possible cause of $T$ wave sensing is described. It is logical that haemodynamic changes can lead to altered repolarisation, and hence $T$ wave changes. Therefore, acute, inappropriate discharges should always lead to suspicion of this disease. Thrombolytic therapy can be indicated if the diagnosis is made. Pulmonary embolism is not exceedingly rare in this population. Our group had one case in 24 patients $^{2}$; others had three of 241 patients, one of 59, and one of $368 . .^{134}$ This is not surprising, as many patients have a low output state, often limiting physical activities. ${ }^{2}$ Finally, an endovascular electrode might serve as a nidus for thrombosis. ${ }^{15}$ In a small series, it was shown that the incidence of floating vegetations on leads is not different between aspirin and anticoagulant treatment. ${ }^{5}$ Further investigation in this domain is needed, as certain subgroups could benefit from aspirin or warfarin treatment.

1 Grimm W, Flores BF, Marchlinski FE. Complications of implantable cardioverter defibrillator therapy: follow-up of 241 patients. PACE 1993;16:218-22.

2 Jordaens L, Vertongen P, Provenier F, Trouerbach JW, Poelaert J, Herregods L. A new transvenous internal cardioverter defibrillator: implantation technique, complications and short term follow-up. Am Heart $\mathcal{f}$ 1995;129: 251-8.

3 Gielchinsky I, Rothbart ST, Parsonnet V, Hussain SM, Fuzesi L, Fontana CA, et al. Safety, feasibility and long term follow-up of a non-thoracotomy defibrillation system in patients with inducible sustained ventricular tachycardia/fibrillation. $\mathcal{F}$ Cardiovasc Surg Torino 1994;35: 111-13.

4 Minten J, Cuypers A, van Binsbergen E, Lindemans F. Medtronic $\$$ Model 7219 Jewel@ PCD® European Clinical Report. Maastricht: Bakken Research Medtronic@ 1995:59.

5 Jung W, Fehske W, Manz M, Pfeiffer D, Tebbenjohanns J, Luderitz $B$. Incidence of floating vegetations on transvenous defibrillation leads: impact of anticoagulant therapy [abstract]. PACE 1993;16:916. 